\title{
Extensional viscosity: a critical discussion ${ }^{\star}$
}

\author{
Christopher J.S. Petrie \\ School of Mechanical $\mathcal{E}$ Systems Engineering, University of Newcastle upon Tyne, \\ Newcastle upon Tyne, NE1 7RU, UK
}

\begin{abstract}
The issue of whether extensional viscosity is a concept that causes more confusion than enlightenment is addressed. This author's view is that misuse of the concept certainly has caused much confusion and, although it is in principle a simple and straightforward idea, it continues to be misused. What is straightforward is the formal definition of extensional viscosity, for steady uniform extensional flow. What gives rise to confusion is the careless use of measurements in flows which are not both steady and spatially uniform.
\end{abstract}

Key words: Trouton, extensional viscosity, extensional flow, history, review PACS: 83.50.Jf, 83.85.Rx, 01.65.+g

\section{Introduction}

The first use of extensional viscosity, under a different name, is that by Trouton [1]. In his 1906 paper he coined the term "coefficient of viscous traction" and his intention was to obtain the viscosity of an incompressible Newtonian fluid. The relationship between the (shear) viscosity, $\eta$, and the coefficient of viscous traction, $\eta_{T}$,

$$
\eta_{T}=3 \eta
$$

was obtained by resolving stresses in appropriate directions, in effect using the tensorial nature of stress and rate of strain. This might be seen as an application of the principle of material objectivity (which was enunciated nearly

ऋ Developed from part of the talk delivered at the INNFM conference, Lake Vyrnwy, Wales in March 2005 with the title "Ninety-nine years of extensional flow".

Email address: Chris.Petrie@Newcastle.ac.uk (Christopher J.S. Petrie). 
50 years later). An historical account of some aspects of extensional flows, including a fuller account of Trouton's work, is given in a companion paper $[2]$.

In this paper we review issues arising from the practical problem that viscosity (in shear or extension) is a material property defined for steady, spatially uniform flows while for practical applications where extensional viscosity is important, flows are never steady and spatially uniform. This has lead to use of the concept of a "transient extensional viscosity" and use of a variety of approximations and averaging techniques.

To anticipate the main conclusion of this paper, we remark here that the use of the term "transient extensional viscosity" or its equivalent is fraught with danger and has led to confusion on a number of occasions. Unless the term is used in a very clear and restricted way, it should not be used at all. The reasons for this conclusion are set out below.

Another issue concerns the connection between extensional and shear flow properties. The "Trouton ratio", defined as $\eta_{T} / \eta$, is 3 for a Newtonian fluid and there are corresponding ratios for biaxial extensional flows (Section 3). For viscoelastic fluids this result will generally hold in the limiting case of very small rates of strain, but otherwise we can say nothing without a specific constitutive equation.

\section{Uniaxial extension}

We consider extensional flows which we define here as having a diagonal rate of strain tensor, D, and a diagonal stress tensor, $\boldsymbol{\sigma}$. A formal definition of "tensile viscosity" is given in the 1984 report of Society of Rheology's second committee on rheological nomenclature [3] reprinted with revisions ten years later [4]. "Tensile viscosity" is the quantity also referred to as "elongational viscosity" and "uniaxial extensional viscosity" and is the same as Trouton's "coefficient of viscous traction". The phrase "extensional viscosity", without further qualification, might refer to any or all of the viscosity functions that could be defined for a steady uniform extensional flow. We shall return briefly to the topic of biaxial and general extensional flows later (Section 3).

The formal definition of "tensile viscosity" is as follows: A material is subjected to homogeneous simple extension, i.e. to a flow which is spatially uniform, with constant rate of strain, $D_{11}=\dot{\varepsilon}$, in the $x_{1}$-direction and $D_{22}=D_{33}=-\frac{1}{2} \dot{\varepsilon}$ in every direction perpendicular to the $x_{1}$-axis. The ratio of "net tensile stress", $\sigma_{E} \equiv \sigma_{11}-\sigma_{22}$, to rate of strain is monitored as a function of time and the 
"tensile viscosity", $\eta_{T}$ is defined as

$$
\eta_{T}(\dot{\varepsilon})=\lim _{t \rightarrow \infty}\left[\frac{\sigma_{E}(t, \dot{\varepsilon})}{\dot{\varepsilon}}\right] .
$$

The notation $\eta_{T}$ is used widely in honour of Trouton, instead of $\eta_{E}$ which is used in the official definition [3], and the tensile or elongational viscosity may be called the "Trouton viscosity".

This definition, of course, says nothing about methods of, or even the feasibility of, experimental realization. It requires that, for steady extension, the tensile stress tends to a constant value (dependent only on the rate of strain, $\dot{\varepsilon}$ ). In practice it also requires that the stress settles down to this constant value within the time of the experiment. Hence if there is no observed steady stress attained within the time of the experiment, there is no elongational viscosity. This is true, in a very obvious way, if we consider an elastic solid and also for the well-known case of an upper convected Maxwell model at rates of strain above a critical value [36, Section 2.2].

A more important practical problem is that a simple extensional flow cannot be realized in a way that is steady both in the Eulerian and the Lagrangean sense (except at a point - a stagnation point). We are faced then either with

(a) experiments that are steady in the laboratory frame of reference but in which the material experiences a changing strain rate, such as fibre spinning, contraction flow or opposed jets,

or with

(b) experiments with a constant strain rate but changing position of the material (except at a stagnation point), such as the tensile test (stretching of a cylinder).

In either case, the experiment is necessarily of limited duration. For some experimental arrangements there is an obvious limitation due to the stretching of the sample to the maximum length which can be accommodated in the apparatus (or the laboratory). The less avoidable limitation is that of a minimum final sample radius and hence a maximum extensional strain, beyond which the sample becomes irregular in shape or breaks. There is therefore a serious question about whether steady flow can practically be achieved.

We should acknowledge here that things are not necessarily quite as bad as we are suggesting here. It is possible that in a fibre spinning experiment the rate of strain is (nearly) constant over a large part of the spinline. In a similar way, it is possible that in a tensile test the cylinder remains a cylinder over most of its length. However we cannot guarantee this a priori; the issue is one 
of the controllability of the flow to which we return briefly below (Section $7)$.

Of course, from a practical point of view, if steady extensional flow is rarely obtained, the tensile viscosity is not the most useful thing to know about the material. Rather we shall need to know something of the behaviour of the material in transient extensional flows and in flows with deformation that involves both extension and shearing. This will mean either that we need a constitutive equation applicable to the material under all the relevant flow conditions or that we need an empirical test that allows us to predict the material behaviour for a specific application.

Finally we note that, if surface tension is significant, we need to be clear that the "net tensile stress" $\sigma_{E}$ in our definition, Eqn. (2), is the measured stress corrected for surface tension according to

$$
\sigma_{E}=\frac{\text { Applied force }}{\text { Area }}-\frac{\text { Coefficient of surface tension }}{\text { Radius }} .
$$

We also need to be clear that the use of $\sigma_{E} \equiv \sigma_{11}-\sigma_{22}$ is, in any case, valid only for incompressible fluids. The normal stress difference $\sigma_{11}-\sigma_{22}$ is used in order to avoid any dependence on hydrostatic pressure. This matter is discussed further elsewhere [5,6]. For simplicity, our discussion of other extensional flows and of the various extensional viscosities that are defined in Eqns. (6), (8), (9), (12) and (13) below (Section 3), refers to incompressible fluids in the absence of surface tension.

\section{General (biaxial) extension}

As well as uniaxial extension, we may consider equibiaxial extension,

$$
D_{11}=D_{22}=\dot{\varepsilon} ; \quad D_{33}=-2 \dot{\varepsilon}
$$

and planar extension,

$$
D_{11}=\dot{\varepsilon} ; \quad D_{22}=0 ; \quad D_{33}=-\dot{\varepsilon} .
$$

The equibiaxial extensional viscosity $[3,4]$ is defined by

$$
\eta_{B}(\dot{\varepsilon})=\frac{\sigma_{11}-\sigma_{33}}{\dot{\varepsilon}}
$$


and for a Newtonian fluid

$$
\eta_{B}=6 \eta_{0}
$$

Equibiaxial extension is kinematically the reverse of uniaxial extension, but significantly different in terms of the effect of the flow in tending to align long molecules or fibres. The idea that the functions $\eta_{T}(\dot{\varepsilon})$ and $\eta_{B}(\dot{\varepsilon})$ may be regarded as the same function, for positive and negative values of the rate of strain in the direction of the axis of symmetry, is not a particularly helpful one since there is no reason at all for supposing that the values of this function over the two ranges of values of its argument are in any way connected (except that both show Newtonian behaviour in the limiting case of small rate of strain). The only way one may connect the two functions is through a constitutive equation.

Planar extension is sometimes referred to as "pure shear" but it must be clearly understood that it is qualitatively different from simple shear, being irrotational (relative to the usual fixed axes). A particular point of interest for planar extension is that there are two extensional viscosities, the planar extensional viscosity

$$
\eta_{p}(\dot{\varepsilon})=\frac{\sigma_{11}-\sigma_{33}}{\dot{\varepsilon}},
$$

which refers to the tensile stress required to stretch the material in the $x_{1^{-}}$ direction and a second extensional viscosity or "cross-viscosity"

$$
\eta_{2}^{(0)}(\dot{\varepsilon})=\frac{\sigma_{22}-\sigma_{33}}{\dot{\varepsilon}},
$$

which refers to the tensile stress required to prevent deformation in the neutral direction (the $x_{2}$-direction). The theoretical relations for a Newtonian fluid, with shear viscosity $\eta_{0}$, are

$$
\eta_{p}=4 \eta_{0} ; \quad \eta_{2}^{(0)}=2 \eta_{0} .
$$

The notation $\eta_{2}^{(0)}$ is a simple example of the notation [3,4,7-9] for general extensional flows. If $D_{11}=\dot{\varepsilon}$ is the largest (positive) rate of strain then we may define $m$ such that $D_{22}=m \dot{\varepsilon}$, with $-0.5 \leq m \leq 1$ and then

$$
D_{11}=\dot{\varepsilon} ; \quad D_{22}=m \dot{\varepsilon} ; \quad D_{33}=-(1+m) \dot{\varepsilon}
$$

for an incompressible fluid. The parameter $m$, which we take to be independent of time, describes the geometry of the extensional flow, with $m=1$ for 
equibiaxial extension, $m=0$ for planar extension, $m=-\frac{1}{2}$ for uniaxial extension and $-\frac{1}{2} \leq m \leq 1$ in general. The two extensional viscosities for this general flow are

$$
\eta_{1}^{(m)}(\dot{\varepsilon})=\frac{\sigma_{11}-\sigma_{33}}{\dot{\varepsilon}}
$$

and

$$
\eta_{2}^{(m)}(\dot{\varepsilon})=\frac{\sigma_{22}-\sigma_{33}}{\dot{\varepsilon}} .
$$

These are equal to one another for equibiaxial extension and the second is zero for uniaxial extension while for all other extensional flows we have the two physical quantities which we may try to measure. As well as for the three standard cases, $m=-\frac{1}{2}, m=0$ and $m=1$, experiments have been carried out by Demarmels and Meissner [10,11] for other extensional flows. For example, $m=+\frac{1}{2}$ has been referred to as "ellipsoidal extension" [12] and may be visualized as an unequal biaxial extension, with stretching at rates $\dot{\varepsilon}$ and $\frac{1}{2} \dot{\varepsilon}$ in two perpendicular directions.

\section{Experimental methods and results}

The basic experimental arrangements are discussed in detail in a variety of books and papers dealing with polymer melts [13,14], polymer solutions [15], contraction flows [16,17], elongational rheometers [18] and filament stretching [19-21].

Here we omit most of the detail and catalogue them in a similar way to James \& Walters [15] and this author's earlier paper [6]:

(1) The tensile test (Trouton's method (A), [2, Section 2.1]) and the filament stretching rheometer $[13,14,22,15,18-21]$.

(2) Fibre spinning (Trouton's method (C), [2, Section 2.1]) [13,14,22,15,18].

(3) Stagnation point flows (four-roll mill, opposed jets) [22,15,23,18,24].

(4) Converging and contraction flows [14,22,15-17].

The tensile test appears different when applied to polymer melts (and other highly viscous materials) [13], where the aim is the extend a cylinder that is as uniform as possible, and when applied to polymer solutions and other mobile liquids [18,20], where measurements are made at the central plane of symmetry and end effects cannot be ignored except at that plane. The difference arises from the different relative magnitudes of forces (in particular 
viscous and capillary forces) and is connected to the issue of controllability. Stagnation point flows are important for realizing planar extension and this has found application at the single-molecule level in elegant experiments on visualizing the unravelling of DNA molecules (see the review by Shaqfeh [24]).

Kinematically the conventional spinning experiment is the same as flows such as Fano flow, apart from different initial conditions and these, like the stagnation point flows, have the major disadvantage that the flow is not materially steady. This is a disadvantage if we want to talk about a material property, but may in fact be an advantage if we want to investigate fluid rheology in situations of practical relevance which are rarely materially steady. Both parts of this remark apply even more strongly with reference to the use of converging flows to estimate extensional properties. It should also be noted that, as can be seen by comparing the chapters by Binding [16] and Gibson [17] that there is not yet universal agreement on how to infer extensional viscosity from converging flow.

The variety of results that can be obtained is well illustrated by the various "round robin" series of experiments referred to in the companion paper $[2$, Section 3]. The "M1" series of experiments are reported in a special issue of this journal [25] and summarized in the well-known Fig. 1 [15]. Use of different techniques involving different flow histories gives values which, when presented as "transient extensional viscosity" as a function of rate of strain, are not consistent one with another, as Fig. 1 clearly shows. This presents, qualitatively, results from ten sets of experiments on a graph of extensional viscosity against rate of extensional strain. For all rates of strain between about $1 \mathrm{~s}^{-1}$ and $50 \mathrm{~s}^{-1}$, a range of values of extensional viscosity from different experiments from about 20 to $10,000 \mathrm{~Pa} \mathrm{~s}$ is reported. The corresponding Trouton ratios are between 6 and 3,000. There is a similar result for test fluid, "A1" [26]. Walters [27, pp.20-21] comments on this situation in a critical but positive way.

In their review, James \& Walters [15] report on the success (to a limited extent) of modelling the different flows in the series of techniques used for the "M1" series of experiments, using the Oldroyd model [29-31] and the KBKZ model $[31,32]$ (see also [33]). This gets to the heart of the matter, that most methods of estimating the extensional viscosity of mobile liquids rely on a constitutive assumption in order to be interpreted correctly. It is important to note, however, that use of a constitutive assumption does not in fact allow us to talk sensibly about an extensional viscosity function for all of the variety of extensional flow experiments that may be used. The experiments represented in Fig. 1 (labeled by the names of the authors) are as follows: 


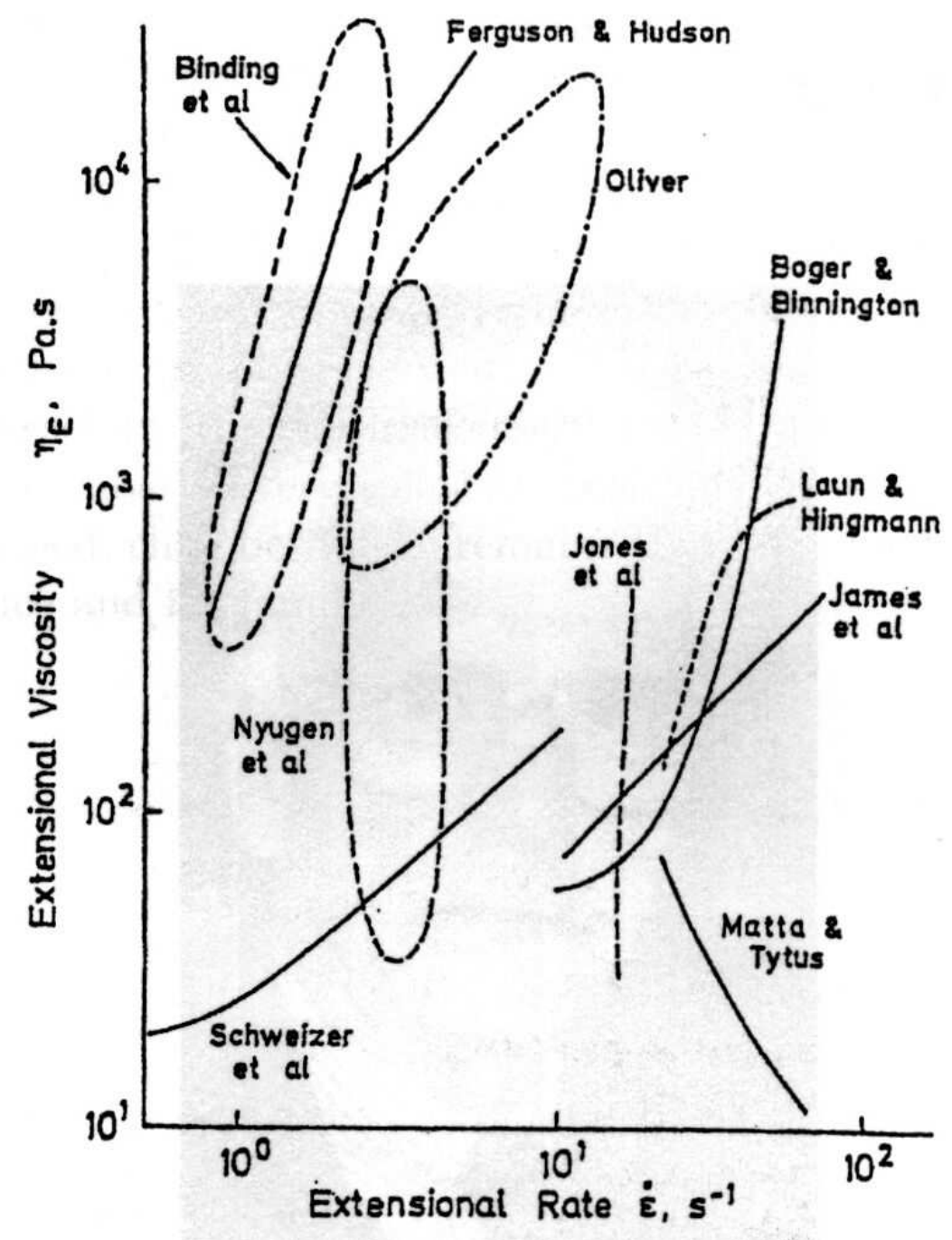

Fig. 1. The "M1" muddle (James \& Walters [15])

Binding et al.

spinline rheometer

Boger \& Binnington

contraction flow

Ferguson \& Hudson

spinline rheometer

James et al.

converging flow rheometer

Jones et al.

pendant drop (filament stretching)

Laun \& Hingmann

opposed jets

Matta \& Tytus

falling weight (filament stretching)

Nguyen et al.

filament stretching

Oliver

horizontal jet (spinning)

Schweizer et al.

opposed jets (stagnation flow) 
Reference to the original paper shows that Binding et al. [28] also obtained results from an open syphon (Fano flow) which were broadly similar to those from the spinline rheometer and from contraction flows, which gave results off the top of the diagram as plotted (apparent viscosity values from $10^{4}$ up to $10^{8}$ (Pa.s.) at strain rates of around $10 \mathrm{~s}^{-1}$.

The point is that, if we did discover the constitutive equation that perfectly modelled the rheology of the fluid "M1", or if we carried out the experiments perfectly, we should get essentially the same picture as Fig. 1 if we try to present the results of the experiments as graphs of "transient extensional viscosity" against rate of strain. Each experiment is showing the response of the fluid to a different extensional flow (with different flow history) and each is correct.

\section{Separation of elastic and viscous effects}

Interest in separating elastic and viscous effects can be found more than a hundred years ago. Trouton [34] addresses this issue and makes the suggestion that elastic and viscous responses might be found together only in mixtures where one component is viscous and one elastic. He uses an experiment on a pure substance (sodium stearate) to show that the suggestion is false - the pure substance shows a combination of elastic and viscous behaviour in the same way as mixtures such as pitch and glass. We have noted in the companion paper [2, Section 2] a number of other papers which address the same issue.

The idea of separating elastic and viscous effects is particularly interesting in some early work of Vinogradov [35]. Vinogradov defined a "transient extensional viscosity" as the ratio of extensional stress to rate of viscous strain, i.e. the rate of increase of non-recoverable strain. Now when steady flow is achieved, the recoverable strain becomes constant since removing the applied stress at any time after steady flow is achieved will lead to the same recoil (strain recovery). Hence the rate of strain and the rate of viscous strain are equal in steady flow and there are no potentially confusing alternative definitions for the "tensile viscosity" (which by definition is obtained from steady flow).

There is the possibility of confusion in discussing transient extensional flow [36, Section 2.2]. For example, Vinogradov's graphs [35, Fig. 10] are sometimes carelessly interpreted as evidence for stress overshoot (stress growing to a value exceeding its steady state value) in the start-up of extension at constant rate of strain. It is not the stress that overshoots; the maximum in the graph occurs because the rate of viscous strain is less than its steady value while the elastic strain is still building up. In order to clarify this we present the results of 

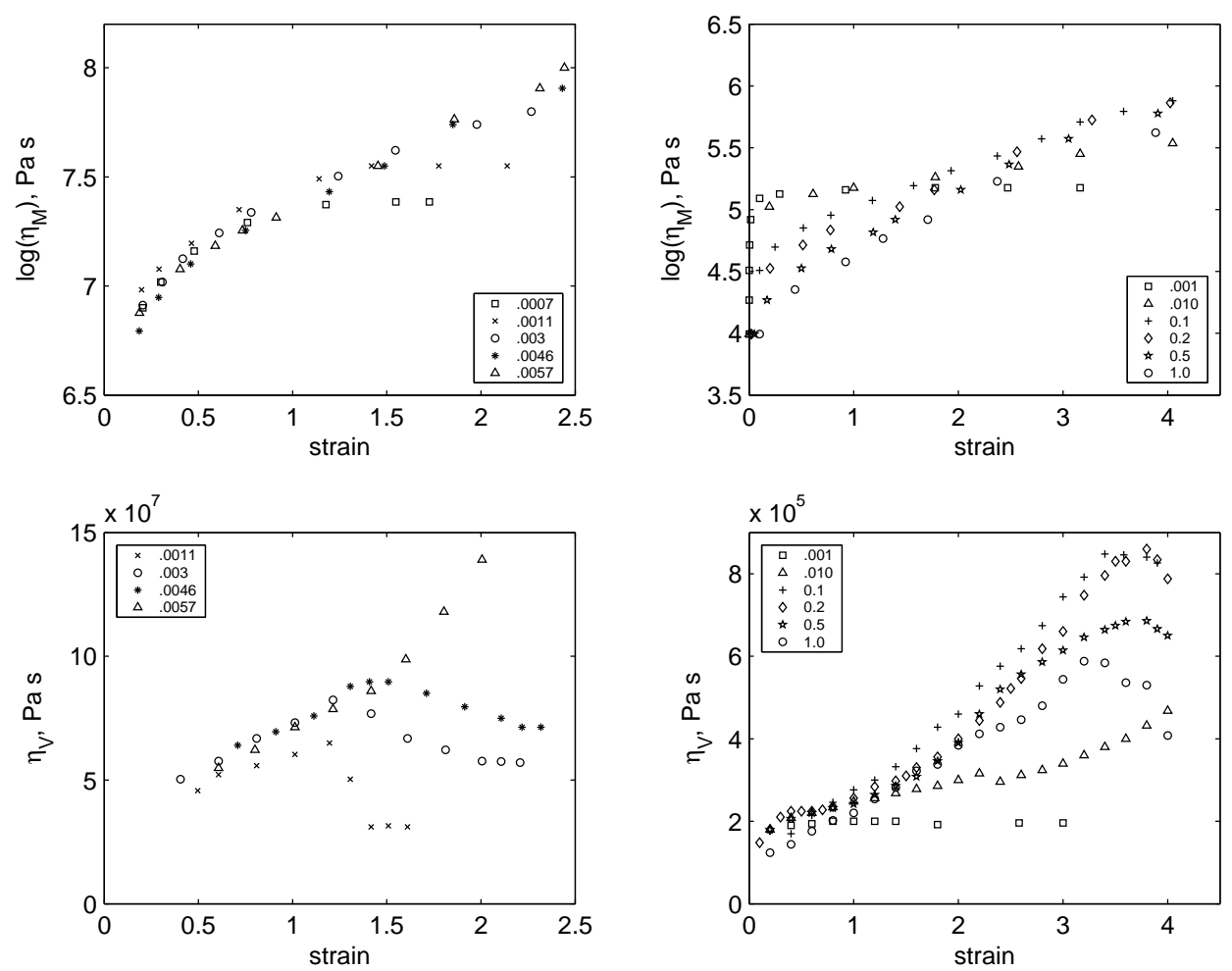

Fig. 2. "Transient extensional viscosity" functions: results of Vinogradov [35] (left-hand graphs) and Meissner [37] (right-hand graphs) presented as $\log \left(\eta_{V}\right)$ and $\eta_{M}$ as functions of strain; based on [38]. (The legends in each figure give rates of strain, $\mathrm{s}^{-1}$, for different experiments.)

Meissner [37] and Vinogradov [35] each plotted in the two ways used by the two authors. The transient results are given as

(a) stress divided by rate of strain - this is Meissner's "stressing viscosity", for which we use the symbol $\eta_{M}$

or as

(b) stress divided by rate of viscous strain - this is Vinogradov's "longitudinal viscosity", for which we use the symbol $\eta_{V}$.

Plotting the results from both laboratories in both ways, Fig. 2, shows that both are obtaining qualitatively similar results [38].

The two upper figures show $\log \eta_{M}$ plotted against strain for the Vinogradov data (left) and the Meissner data (right, as published). The two lower figures show $\eta_{V}$ plotted against strain for the Vinogradov data (left, as published) and the Meissner data (right). The magnitudes of the viscosities differ because different polymers were used - the point is to note the qualitative similarity between the results from the two laboratories when presented in the same way. There is no evidence of overshoot for $\eta_{M}$ from either set of data and 
clear evidence of overshoot in the (less common) $\eta_{V}$ for both sets of data. More recently Hassager and colleagues [39] report definite overshoot in the transient extensional viscosity (as commonly defined, $\eta_{M}$ ) for LDPE at high rates of strain.

Note that we use strain as the abscissa in these graphs, as is usual for "transient extensional viscosity" from constant rate of strain experiments; it is perhaps helpful if we regard the graphs as stress growth curves in which we plot stress (scaled by dividing by rate of strain or rate of viscous strain) against dimensionless time (scaled by multiplying by rate of strain, giving the total strain). This helps to bring together results for different dates of strain. See also the discussion below (Section 6.4) on strain and rate of strain dependence of extensional viscosity.

There is no doubt that, as a qualitative way of thinking about material behaviour, the separation of viscous and elastic responses is helpful; however there is also no doubt that linear superposition of elastic and viscous strain is not in general possible. The non-linearity of the material responses with which we are faced means that, for example, if we double the strain, we do not necessarily double the elastic strain. This can be verified by considering the result of calculations done for a simple model viscoelastic fluid [40] (a Jeffreys fluid or Oldroyd fluid B). Elastic strain (recoverable strain, i.e. the actual strain recovered when the applied stress is removed) is calculated for uniaxial extension at constant rate of strain. The viscous strain is obtained by subtracting the elastic strain from the total strain. There is clear evidence of non-linearity in the fact that the proportion of the strain which is elastic depends on the rate of strain.

An alternative to separating the strain into elastic (recoverable) and viscous (non-recoverable) parts is similarly to separate the stress. This is associated with the use of the filament stretching rheometer by McKinley [41] and Sridhar $[42,43]$ to study of stress relaxation at the end of an extensional flow experiment. This gives an elastic response of the material, since when there is no motion there is no viscous response. Of course it is not quite as simple as that since there is no stress relaxation for a purely elastic material and so one is clearly measuring a property of a viscoelastic material. Again the non-linearity of the material means that the separation into elastic and viscous stresses is not achievable in the way it can be done for a linear viscoelastic material. The two ways of proceeding (in terms of strain or stress) correspond to linear models with springs and dashpots in series or in parallel. 


\section{Some theoretical considerations}

\subsection{Inelastic fluids}

Reiner [44], unlike others in the 1930s and 1940s, gave Trouton credit for both the theoretical deduction and the experimental verification of the value 3 for the Trouton ratio. He pointed out that Trouton's result is not correct if compressibility of the fluid and bulk (or volume) viscosity are taken into consideration. He went on to discuss the "generalized Newtonian liquid", by which he meant what we would now call the Reiner-Rivlin fluid in which stress is the most general isotropic function of rate of strain alone,

$$
\boldsymbol{\sigma}=f_{1} \mathbf{I}+f_{2} \mathbf{D}+f_{3} \mathbf{D}^{2}
$$

in which $f_{1}, f_{2}$ and $f_{3}$ are functions only of the invariants of $\mathbf{D}$. The first invariant, $I_{\mathbf{D}}=\operatorname{tr}(\mathbf{D})$, is zero for incompressible fluids and the third, $I I I_{\mathbf{D}}=$ $\operatorname{det}(\mathbf{D})$, is zero for planar extension and for simple shear but non-zero for uniaxial extension and other more general extensional flows. For the second invariant we take here, for simplicity, $I I_{\mathbf{D}}=\frac{1}{2} \operatorname{tr}\left(\mathbf{D}^{2}\right)$.

We may show that the Trouton ratio of 3 is not obtained for every incompressible Reiner-Rivlin fluid, Eqn. (14) with $f_{i}\left(I I_{\mathbf{D}}, I I I_{\mathbf{D}}\right)$. In simple shear, with shear rate $\dot{\kappa}$ we have a variable viscosity

$$
\eta=\frac{1}{2} f_{2}\left(\frac{1}{4} \dot{\kappa}^{2}, 0\right)
$$

while in uniaxial extension

$$
\eta_{T}=\frac{3}{2} f_{2}\left(\frac{3}{4} \dot{\varepsilon}^{2}, \frac{1}{4} \dot{\varepsilon}^{3}\right)+\frac{3}{4} \dot{\varepsilon} f_{3}\left(\frac{3}{4} \dot{\varepsilon}^{2}, \frac{1}{4} \dot{\varepsilon}^{3}\right) .
$$

Hence the Trouton ratio of 3, Eqn. (1), applies for any purely viscous fluid in which stress is equal to rate of strain multiplied by a viscosity that depends on the second invariant of the rate of strain. The comparison of extension and shear is carried out for $\dot{\varepsilon}=\dot{\kappa} / \sqrt{3}$. The Trouton ratio is not 3 either where the viscosity function, $f_{2}$, in Eqn. (14) depends on the third invariant of the rate of strain or where the additional viscosity function, $f_{3}$, is non-zero.

If we take a rather pragmatic approach and fit experimental data using, for example, power-law expressions for viscosity in simple shear and uniaxial extension [17] this may be useful as a way of summarizing data and making predictions for flows very similar to either simple shear or uniaxial extension. 
The use of superposition for other flows [22, Section 2.4.5] has been suggested, but this approach will not give correct results for other extensional flows (e.g. planar or equibiaxial). Probably if this approach is to be used in complex flows, these should be axisymmetric flows such as converging or contraction flows. The superposition is unlikely to find a formal mathematical justification, given the non-linearity of the materials to which we want to apply it. We find a discussion of these ideas and their computational application by Debbaut and Crochet [45] which points out that such purely viscous models are useless in planar flows (in connection with distinguishing shear and extensional behaviour). Their idea is to try and discover the consequences of different Trouton ratios for the simplest model (i.e. the purely viscous fluid).

\subsection{Viscoelastic fluids}

Coleman and Noll [46] proved that for any "simple fluid" (in which the stress can depend on the deformation history of the the material) in a simple extensional flow (steady rectilinear extension) the equation,

$$
\boldsymbol{\sigma}=g \mathbf{I}+h \mathbf{D}+\ell \mathbf{D}^{2},
$$

holds, where $g, h$ and $\ell$ are functions of the invariants of $D$. It is important to distinguish clearly between this result, Eqn. (17), which applies to viscoelastic fluids, but only for steady extensional flow, and Eqn. (14) which is a purely viscous constitutive equation applying to any flow of a material described by this constitutive equation. The result, Eqn. (17), is important, but often of limited practical applicability and does not help us to make connections between behaviour in shear and in extension unless we have a specific constitutive equation. For example, for the upper convected Maxwell model with relaxation time $\lambda$ and viscosity $\eta$, we may deduce [5] that

$$
\begin{aligned}
h & =\frac{2 \eta}{1-4 \lambda^{2} I I_{\mathbf{D}}-8 \lambda^{2} I I I_{\mathbf{D}}}, \\
\ell & =\frac{4 \eta \lambda}{1-4 \lambda^{2} I I_{\mathbf{D}}-8 \lambda^{2} I I I_{\mathbf{D}}} .
\end{aligned}
$$

It is straightforward to deduce from this pair of equations, the expressions for uniaxial, planar and equibiaxial extensional viscosities for the upper convected Maxwell model.

However a further warning is necessary. Not only can we not use Eqn. (17) to deduce behaviour in uniaxial extension from behaviour in simple shear, but we cannot even deduce behaviour in other extensional flows from that in uniaxial extension. This follows from the fact that each type of extensional flow gives a 


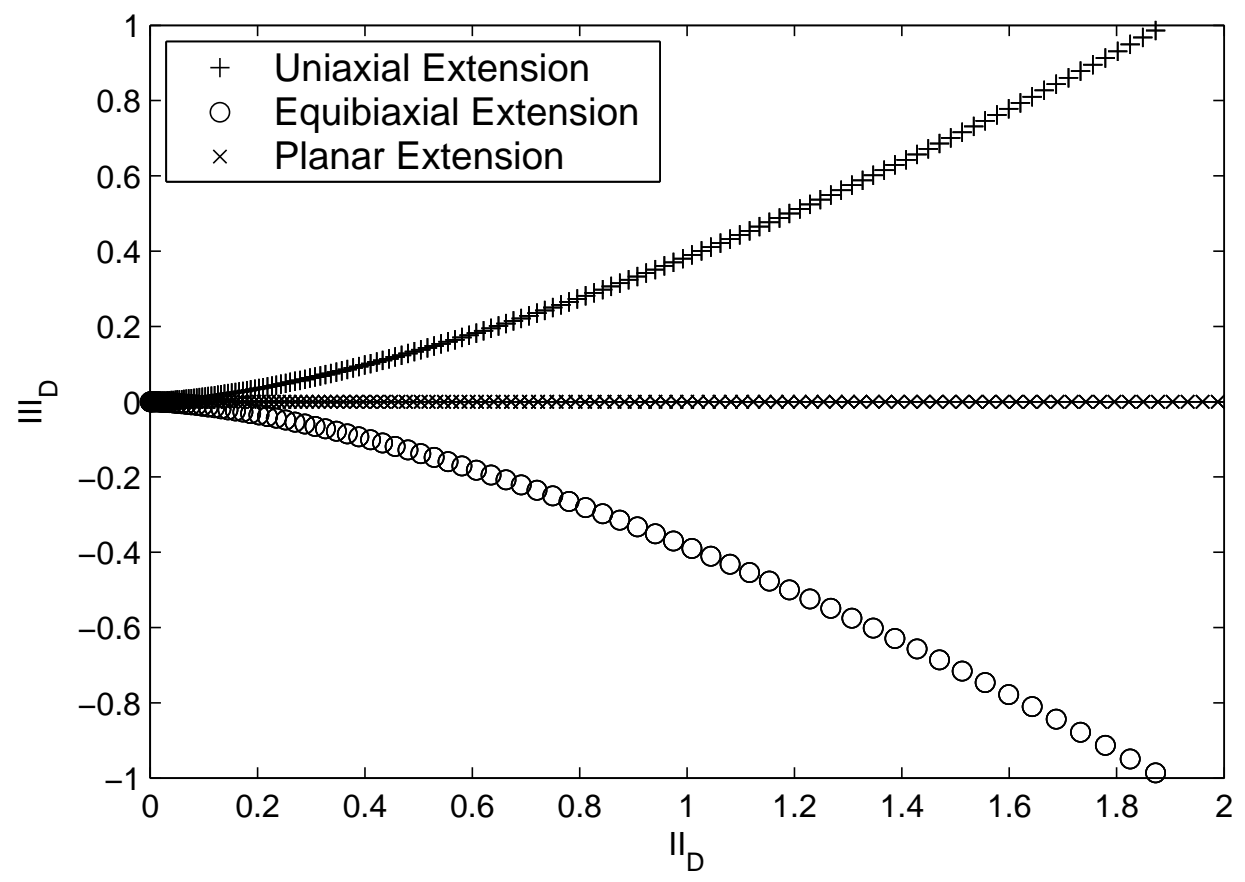

Fig. 3. Relation between the invariants of rate of strain for the standard extensional flows.

specific relationship between the two invariants on which the functions $h$ and $\ell$ depend. This may be visualized by saying that each of the three standard extensional flows corresponds to a different path in a plot of $I I_{\mathbf{D}}$ against $I I I_{\mathbf{D}}$, Fig. 3.

Of course, if we have a constitutive equation whose parameters may all be calculated from a set of measurements in any one or two flows, we may then predict behaviour in all other flows. Such predictions will be useful tests of the correctness of the constitutive equation, but there is no way of making such predictions without a constitutive equation.

\section{3 "Transient extensional viscosity"}

We saw above (Section 4) that different extensional flow experiments give us different functions for "transient extensional viscosity". We might then note that the results for similar types of experiment are grouped together, roughly speaking, in Fig. 1. Unfortunately we can demonstrate that even in an idealized situation this may not be the case.

We use a FENE-P dumb-bell model to simulate fibre spinning and presenting the results as a graph of "transient extensional viscosity" against rate of strain. Different values of the applied force and different positions along the spinline give results throughout the shaded region shown in Fig. 4 [6]. The initial extra- 


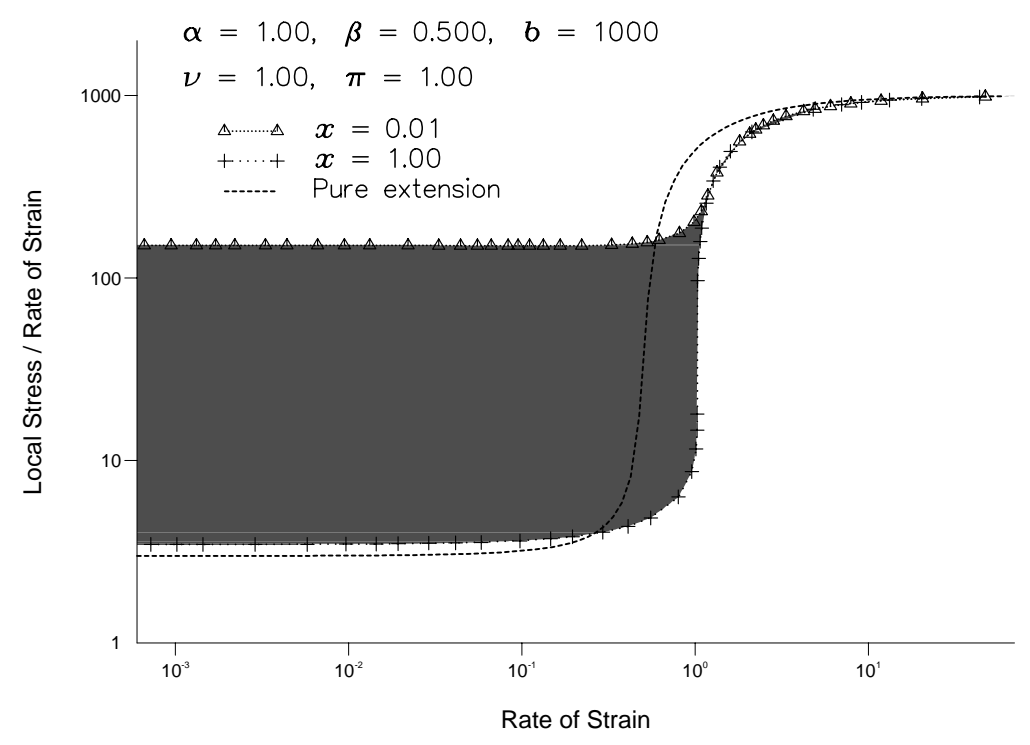

Fig. 4. A range of apparent extensional viscosity behaviour in spinning [6, Fig 1b]. The dimensionless "Local stress/rate of strain" is scaled by the zero-shear rate viscosity (sum of polymer and solvent contributions) and the dimensionless rate of strain is scaled by the relaxation time. $\alpha$ is the Deborah number (based on initial velocity and spinline length), $\beta$ the dimensionless polymer contribution to the viscosity, $b$ the finite extensibility parameter and $\nu$ and $\pi$ are the initial values of extra-stress components. The boundaries of the shaded area correspond to distances $x$ along the spinline of $1 \%$ and $100 \%$ of the total spinline length.

stress values are not varied so this demonstration does not even include the possible effects of different deformation histories prior to the spinline.

The conclusion from this set of calculations is that, for this model, at least at low rates of strain (dimensionless rate of strain less than 1 for Fig. 4) it is not possible to obtain a unique "transient extensional viscosity" as a function of rate of strain from spinning experiments and the idea is not even a useful approximation in this situation. For rates of strain above the dimensionless value of 1 there may be some sense in defining what one might call a "spinning viscosity" but this conclusion is, so far, only supported by the calculations for one particular constitutive equation.

\subsection{Strain or rate of strain dependence}

One response to the difficulty in getting mutually consistent results from different experiments is to suggest that the "transient extensional viscosity" should be viewed as functions of strain rather than functions of rate of strain. This has allowed a number of authors to present data that look more consistent. 
It may also seem to make sense when one considers that material properties are influenced by the microstructure (e.g. macromolecular stretching and alignment) in a sample of material.

However strain in a liquid is somewhat arbitrary, since strain must be measured relative to a reference configuration and there is no unique reference configuration for a liquid. Typically the strain in a fibre relative to its configuration at the die from which it is extruded is used. There are two obvious ways in which this will be an inadequate measure of the microstructure of the material. One is that the microstructure will, for many materials, tend to revert to an isotropic relaxed state. The other is that both orientation and stretching have upper limits while the measured strain does not. Both of these will mean that the strain overestimates the microstructural changes. Measurement of recoverable strain may be illuminating here but this will not help our understanding as much as analysis using an appropriate constitutive equation.

Note that the use of strain as the abscissa in Fig. 2 above is not relaated to this issue, as discussed above (Section 5).

\section{Concluding remarks}

One forgotten idea, from the heyday of continuum mechanics, is that of the controllability of a flow. This seems to be a significant issue since none of the flows we have discussed is controllable in the sense that the experimenter prescribes the flow field in a definite way. In filament stretching one relies on a balance of viscous, elastic and capillary forces to maintain the sample in a (near) cylindrical shape. In spinning the history of the rate of strain is not directly prescribed but depends on the fluid response to the flow (and thus the shape of the fibre being spun may vary with flow rate). In the opposed jet configuration, how much fluid stays for how long near the stagnation point is dependent on the actual flow conditions and material properties. Similarly the flow field in contraction flows is not prescribed a priori by the experimenter. This leaves us at the mercy of the material, particularly when steady flow is not achieved, and one consequence is that a correlation between transient results for different types of experiment that we might obtain for one material would not be likely to hold for any other material.

We reiterate the warning about extensional viscosity: it is fine in theory but is a very dangerous idea in practice, specifically when it is applied when steady flow has not been achieved. The convenience of referring to a "transient extensional viscosity" is almost certainly an oversimplification that is liable to lead to the false notion that one might obtain the same dependence on time for different types of experiment. Even to confine the simplification to one type of 
experiment and to define a "spinning viscosity" may be more than is justified by experimental observation and certainly can be shown to be wrong for some theoretical models.

However, there is no doubt that transient extensional flows are what we have in practical situations, so can we say something slightly more positive?

- One analogy offered by McKinley [47] is from thermodynamics: the difference between path-independent functions (like internal energy or steadystate elongational viscosity) and path-dependent ones (such as work, heat, transient elongational viscosities). Defining path-dependent functions such as work and heat has helped us understand thermodynamics and indeed has been vital to its development.

- Another analogy that may help is with quantities such as the Melt Flow Index, obtained in standardized technical tests. A "transient extensional viscosity", carefully defined for a specific experiment, must surely be at least as useful as the MFI in comparative assessment of fluid properties as far as they are relevant to real flows with extensional deformations taking place for limited times. The analogy with MFI may be better if applied to averaged quantities (such as a "spinning viscosity" based on draw ratio, spinline length, flow rate and applied force but ignoring details of the fibre profile, details which we would need to get local values of stress and rate of strain). The Rheotens [14] equipment offers a technical test using fibre spinning in a prescribed way to obtain a "melt strength" and this is perhaps more appropriate than simplified deductions of some sort of extensional viscosity from spinning.

- In spite of the reservations expressed above (Section 6.4), a measure of strain may be the best readily accessible measure of stretching and even orientation at the microstructural level. Hence, provided the conditions of the experiment are clearly described, it seems sensible to accept that the use of a "transient extensional viscosity" expressed as a function of strain will tell us something about material rheology in extension.

- Use such a function with care - it is a simplification and sometimes will be an over-simplification. The point is well put by Pearson [48]:

"My main message is as simple as yours: if you want to predict flow in all circumstances, you need a REoS [a rheological equation of state or constitutive equation], nothing less. Rheometric functions can be useful in classification and categorisation, involving qualitative statements, and can provide engineering approximations in particular flow fields, but they cannot be inserted in CFD packages."

The author is indebted to a number of friends and colleagues. His interest in extensional flow can be traced back over 40 years to post-doctoral work with Anthony Pearson in 1964 and a sabbatical spent with Morton Denn at the University of Delaware in 1974. The late Georgii Vinogradov supplied data 
and encouragement on more than one occasion. Morton Denn, David James, Gareth McKinley, Anthony Pearson, Roger Tanner and Ken Walters made comments on a draft of this paper, though it is certain that these would not all agree with all that has been written here.

\section{References}

[1] F.T. Trouton, On the coefficient of viscous traction and its relation to that of viscosity Proc. Roy. Soc., A77 (1906) 426-440.

[2] C.J.S. Petrie, One hundred years of extensional flow, J. Non-Newtonian Fluid Mech., This volume (2006).

[3] J.M. Dealy, Official nomenclature for material functions describing the response of a viscoelastic fluid to various shearing and extensional deformations, J. Rheol., 28 (1984) 181-195.

[4] J.M. Dealy, Official nomenclature for material functions describing the response of a viscoelastic fluid to various shearing and extensional deformations. Reprinted version with additions and corrections, J. Rheol., 38 (1994) 179-191.

[5] C.J.S. Petrie, Elongational Flows, Pitman (now Longman), London (1979).

[6] C.J.S. Petrie, Extensional flow - a mathematical perspective, Rheol. Acta, 34 (1995) 12-26.

[7] J. Meissner, S.E. Stephenson, A. Demarmels and P. Portmann, Multiaxial elongational flows of polymer melts - classification and experimental realization, J. Non-Newtonian Fluid Mech., 11 (1982) 221-237.

[8] C.J.S. Petrie, Extensional flows of Oldroyd fluids, J. Non-Newtonian Fluid Mech., 14 (1984) 189-202.

[9] C.J.S. Petrie, Some asymptotic results for planar extension, J. Non-Newtonian Fluid Mech., 34 (1990) 37-62.

[10] A. Demarmels and J. Meissner, Multiaxial elongations of polyisobutylene with various and changing strain rate ratios, Rheol. Acta, 24 (1985) 253-259.

[11] A. Demarmels and J. Meissner, Multiaxial elongations of polyisobutylene and the predictions of several network theories, Coll. Polym. Sci., 264 (1986) 829846.

[12] M.H. Wagner and A. Demarmels, A constitutive analysis of extensional flows of polyisobutylene, J. Rheol., 34 (1990) 943-958.

[13] J. Meissner, Rheometry of polymer melts, Ann. Rev. Fluid Mech., 17 (1985) 45-64.

[14] J.M. Dealy, Rheometers for Molten Plastics, Van Nostrand Reinhold, New York (1982). 
[15] D.F. James and K. Walters, A critical appraisal of available methods for the measurement of extensional properties of mobile systems, in A.A. Collyer (Ed.), Techniques of Rheological Measurement, Elsevier, New York (1994) pp.33-53.

[16] D.M. Binding, Contraction flows and new theories for estimating extensional viscosity, in A.A. Collyer (Ed.), Techniques of Rheological Measurement, Elsevier, New York, (1994) pp.1-32.

[17] A.G. Gibson, Converging dies, in A.A. Collyer and D.W. Clegg (Eds.), Rheological Measurement (2nd Edition), Elsevier Applied Science, London (1998) pp.455-491.

[18] R.K. Gupta and T. Sridhar, Elongational rheometers, in A.A. Collyer and D.W. Clegg (Eds.), Rheological Measurement (2nd Edition), Elsevier Applied Science, London (1998) pp.516-549.

[19] S.L. Anna, G.H. McKinley, D.A. Nguyen, T. Sridhar, S.J. Muller, J. Huang and D.F. James, An interlaboratory comparison of measurements from filamentstretching rheometers using common test fluids, J. Rheol., 45 (2001) 83.

[20] G.H. McKinley and T. Sridhar, Filament-stretching rheometry of complex fluids, Ann. Rev. Fluid Mech., 34 (2002) 375-415.

[21] A. Bach, H.K. Rasmussen and O. Hassager, Extensional viscosity for polymer melts measured in the filament stretching rheometer, J. Rheol.47 (2003) 429.

[22] C.W. Macosko, Rheology: Principles, Measurements and Applications, WileyVCH, New York (1994).

[23] G.G. Fuller, Optical Rheometry, Ann. Rev. Fluid Mech., 22 (1990) 387-417.

[24] E.S.G. Shaqfeh, The dynamics of sungle-molecule DNA in flow, J. NonNewtonian Fluid Mech., 130 (2005) 1-28.

[25] Proceedings of an International Conference on Extensional Flow, held at Combloux, France, 20th-23rd March, 1989, J. Non-Newtonian Fluid Mech., 35 (1990) 85-470.

[26] N.E. Hudson and T.E.R. Jones, The A1 project - an overview, J. NonNewtonian Fluid Mech., 46 (1993) 69-88.

[27] K. Walters, Recent developments in rheometry, in R. Keunings and P. Moldenaers (Eds.), Theoretical and Applied Rheology (Proc. XIth Int. Congr. Rheol.), Volume 1, Elsevier, Amsterdam (1992) pp.16-23.

[28] D.M. Binding, D.M. Jones and K. Walters The shear and extensional flow properties of M1, J. Non-Newtonian Fluid Mech., 35 (1990) 121-135.

[29] R.A. Keiller, Modelling of the extensional flow of the M1 fluid with the Oldroyd equation, J. Non-Newtonian Fluid Mech., 42 (1992) 49-64.

[30] D.A. Nguyen, R.K. Gupta and T. Sridhar, Experimental results and constitutive modelling of the extensional flow of M1, J. Non-Newtonian Fluid Mech., 35 (1990) 207-214. 
[31] M.S. Chai and Y.L. Yeow, Modelling of fluid M1 using multiple-relaxation-time constitutive equations, J. Non-Newtonian Fluid Mech., 35 (1990) 459-470.

[32] H.J. Park and E. Mitsoulis, Numerical simulation of circular entry flows of fluid M1 using an integral constitutive equation, J. Non-Newtonian Fluid Mech., 42 (1992) 301-314.

[33] H.J. Park, D. Kim, K.-J. Lee and E. Mitsoulis, Numerical simulation in converging channel flow of the fluid M1 using an integral constitutive equation, J. Non-Newtonian Fluid Mech., 52 (1994) 69-89.

[34] F.T. Trouton and E.S. Andrews, On the viscosity of pitch-like substances, Phil. Mag. (Ser. 6), 7 (1904) 347-355.

[35] G.V. Vinogradov, V.D. Fikhman, B.V. Radushkevich and A.Ya. Malkin, Viscolelastic and relaxation properties of a polystyrene melt in axial extension, J. Polym. Sci. A2, 8 (1970) 657-678.

[36] C.J.S. Petrie and J.M. Dealy, Uniform elongational flow of molten polymers, in G. Astarita, G. Marrucci and L. Nicolais (Eds.), Rheology, Volume 1: Principles (Proceedings of the VIIIth International Congress on Rheology), Plenum Press, New York (1980) pp.171-194.

[37] J. Meissner, Dehnungsverhalten von Polyäthylen-Schmelzen (Elongational properties of polyethylene melts), Rheol. Acta, 10 (1971) 230-242.

[38] G.V. Vinogradov, Personal communication, 1977.

[39] H.K. Rasmussen, J.K. Nielsen, A. Bach and O. Hassager, Viscosity overshoot in the start-up of uniaxial elongation of low density polyethylene melts, J. Rheol., 49 (2005) 369.

[40] C.J.S. Petrie, Recoverable strain in theory, in R. Keunings and P. Moldenaers (Eds.), Theoretical and Applied Rheology (Proc. XIth Int. Congr. Rheol.), Volume 1, Elsevier, Amsterdam, pp.106-108 (1992)

[41] S.H. Spiegelberg and G.H. McKinley, Stress relaxation and elastic decohesion of viscoelastic polymer solutions in extensional flow, J. Non-Newtonian Fluid Mech., 67 (1996) 49-76.

[42] N.V. Orr and T. Sridhar, Stress relaxation in uniaxial extension, J. NonNewtonian Fluid Mech., 67 (1996) 77-103.

[43] N.V. Orr and T. Sridhar, Probing the dynamics of polymer solutions in extensional flow using step strain rate experiments, J. Non-Newtonian Fluid Mech., 82 (1999) 203-232.

[44] M. Reiner, The coefficient of viscous traction, Amer. J. Math., 68 (1946) 672680 .

[45] B. Debbaut and M.J. Crochet, Extensional effects in complex flows, J. NonNewtonian Fluid Mech.30 (1988) 169-184. 
[46] B.D. Coleman and W. Noll, Steady extension of incompressible simple fluids, Phys. Fluids, 5 (1962) 840-843.

[47] G.H. McKinley, Personal communication, 2005.

[48] J.R.A. Pearson, Personal communication, 2005. 